\title{
25 Research Square \\ GUCY2D Gene Loss-of-Function Mutations Responsible for Leber Congenital Amaurosis 1
}

\section{Feng Xue}

School of Medicine, Zhejiang University

Tianying Wei

Zhejiang University School of Medicine

Junhui Sun

Zhejiang University School of Medicine

Yuqin Luo

Zhejiang University School of Medicine

Yanan Huo

Zhejiang University School of Medicine

Ping Yu

Zhejiang University School of Medicine

Jiao Chen

Zhejiang University School of Medicine

Xiaoming Wei

BGT-Wuhan

Ming Qi

Zhejiang University School of Medicine

Yinghui Ye ( $\nabla$ yeyh1999@zju.edu.cn )

Women's Hospital, Zhejiang University School of Medicine https://orcid.org/0000-0001-7520-3871

\section{Research article}

Keywords: Leber congenital amaurosis 1, GUCY2D, catalytic activity, 3',5'-cyclic guanosine monophosphate, guanylate cyclase 1

Posted Date: October 7th, 2019

DOI: https://doi.org/10.21203/rs.2.11649/v2

License: (c) (i) This work is licensed under a Creative Commons Attribution 4.0 International License. Read Full License 


\section{Abstract}

Background: Leber congenital amaurosis (LCA) is a group of severe congenital neurodegenerative diseases. Variants in the guanylate cyclase 2D gene ( GUCY2D ), which encodes guanylate cyclase 1 (ROS-GC1), are associated with LCA1 and account for $6 \%-21 \%$ of all LCA cases. Methods: In this study, one family with LCA1 was recruited from China. A combination of next generation sequencing and Sanger sequencing was used to screen for disease-causing mutations. Additionally, immunohistochemistry and HPLC-coupled tandem mass spectrometry (HPLC-MS/MS) were used to confirm the cellular location and catalytic activity of ROS-GC1 mutants, respectively. Results: We found three novel mutations (c.139_139delC, c.835G>A and c.2783G>A) in the GUCY2D gene. Mutation c.139_139delC results in a truncated protein. Mutations c.835G>A and c.2783G>A exert no effects on cellular location but reduce significantly the catalytic activity of ROS-GC1. Conclusions: Our findings highlight the clinical range of LCA. Moreover, HPLC-MS/MS was used to analyze the concentration of 3',5'-cyclic guanosine monophosphate (cGMP), suggesting that HPLC-MS/MS is an effective alternative method to evaluate the catalytic activity of wild-type and mutant ROS-GC1 Keywords: Leber congenital amaurosis 1, GUCY2D , catalytic activity, 3',5'-cyclic guanosine monophosphate, guanylate cyclase 1

\section{Background}

Leber congenital amaurosis (LCA), accounts for at least $5 \%$ of all retinal dystrophies, and is the earliest and most severe form of all inherited retinal dystrophies [1]. LCA is generally inherited in an autosomal recessive manner [2-4] and characterized by genetic and phenotypic heterogeneity. Currently, mutations in 20 genes are associated with LCA [5]. The distribution of pathogenic genes vary considerably among different populations; however, guanylate cyclase 2D (GUCY2D) is one of the most prevalent LCA gene since GUCY2D mutations account for $6 \%-12 \%$ of LCA cases (LCA1) [4-5].

Guanylate cyclase 1 (ROS-GC1), encoded by GUCY2D, is expressed in rod and cone cells of vertebrae retina and catalyzes the synthesis of 3',5'-cyclic guanosine monophosphate (cGMP). ROS-GC1 is negatively controlled by a $\mathrm{Ca}^{2+}$ feedback loop because a reduction in $\mathrm{Ca}^{2+}$ concentration causes an increase in ROS-GC1 activity. However, the effects of the $\mathrm{Ca}^{2+}$ concentration on ROS-GC1 activity are indirect because this feedback loop is regulated by small $\mathrm{Ca}^{2+}$-binding guanylate cyclase-activating proteins (GCAPs), which interact with overlapping binding sites within the juxtamembrane domain (JMD), kinase homology domain (KHD) and dimerization domain (DD) of ROSGC1. In addition to these latter domains, ROS-GC1 also possesses a leader sequence (LS), an extracellular domain (ECD), a transmembrane domain (TM) and a cyclase catalytic domain (CCD) [6]. Currently, 127 GUCY2D point mutations in different domains of ROS-GC1 cause protein dysfunction and subsequently result in LCA1 [5]. Mutation Pro575Leu located at the JMD domain lacks sensitivity to GCAP1 regulation and induces dysregulation of the $\mathrm{Ca}^{2+}$-sensitive cyclase activation profile. Patients carrying the Pro575Leu mutation suffer from LCA1 by distortion of $\mathrm{Ca}^{2+}-\mathrm{cGMP}$ homeostasis, and these patients require longer periods in the dark to recover [7].

In the present study, targeted-next generation sequencing (NGS) was performed to screen 222 genes that are responsible for 24 kinds of ophthalmic genetic diseases in the proband. After confirming the results by Sanger sequencing, the identity of three novel variants of the GUCY2D gene were found, c.139_139delC (Ala49Profs*36), c.835G >A (Asp279Asn) and c.2783G >A (Gly928Glu). This study probes the pathogenesis of LCA1 and addresses the effects of mutations on ROS-GC1 activity. The three novel mutations may be suitable for LCA1 screening, and HPLC-MS/MS represents a convenient and effective method for cGMP quantification. 


\section{Methods}

\section{LCA patients}

A family with a 5-year-old proband (III-2) and two suspected patients (III-1 and III-3) were recruited for this study. Written informed consent was obtained from each individual to participate in this study. The proband was diagnosed with LCA at Guangdong Zhongshan Hospital, Shanghai General Hospital and the Second Affiliated Hospital of the School of Medicine, Zhejiang University. The pedigree was constructed for the proband based on information provided by the guardians.

\section{DNA isolation and qualification}

Total genomic DNA was extracted using the Relax Gene Blood DNA System (Tiangen, Beijing, China) following the manufacturer's instructions. All DNA was dissolved in sterilized double-distilled water and kept at $-20^{\circ} \mathrm{C}$ until assayed.

One percent agarose gels were used to monitor DNA degradation and contamination. All DNA samples were examined for protein contamination (as indicated by the $A_{260} / A_{280}$ ratio) and reagent contamination (indicated by the $A_{260} / A_{230}$ ratio) with a NanoDrop ND 1000 spectrophotometer (NanoDrop, Wilmington, DE, USA).

\section{Targeted-Next generation sequencing (NGS)}

DNA samples obtained from the proband were sequenced using targeted-NGS. A customized Sequence Capture 2.1M Human Array from Roche NimbleGen (NimbleGen, Madison, USA) was designed to capture 3093 exons (including 100 bp regions that flanked the exons) from 222 genes known to be associated with common genetic diseases, including retinitis pigmentosa, Waardenburg syndrome, $\mathrm{X}$-linked juvenile retinoschisis, crystalline retinitis pigmentosa, albinism, LCA, Bardet Biedl syndrome and cone-rod dystrophy (Additional Table 1). The procedure for the preparation of the libraries was consistent with standard protocols published previously [8]. The data of targeted-NGS assay was analyzed by Illumina basecalling Software 1.7.

\section{Mutation validation by Sanger sequencing}

Sanger sequencing was used to validate candidate variants identified by NGS. Primers of the GUCY2D gene (NG_009092.1) used in Sanger sequencing were designed by Primer-BLAST (http://www.ncbi.nlm.nih.gov/tools/ primer-blast/) and synthesized by Sangon Biotech (Shanghai, China) (Table 1). All amplifications were examined by electrophoresis using $2 \%$ agarose gels and sequenced by BioSune Biotechnology Co., Ltd. (Shanghai, China). The sequencing results were further compared and analyzed by Mutation Surveyor [9].

\section{Construction of wild-type (wt) and mutant human ROS-GC1 recombinant plasmids}


The cDNA of human ROS-GC1 was obtained from Gene Copoeia (EX-Z0715-M98). Primers F and R containing Xhol and Agel restriction sites were used to amplify ROS-GC1 (Table 2). The pEGFP-N1 vector was digested with Xhol and Agel restriction enzymes. PCR amplification product was sub-cloned into pEGFP-N1 using ClonExpress (Vazyme, Nanjing, China) to give the recombinant plasmid pEGFP-GC1.

Site-directed mutagenesis PCR was used to construct the ROS-GC1 mutants. Primers used in site-directed mutagenesis PCR are shown in Table 2. Each mutant was achieved by two-step PCRs using pEGFP-GC1 as the template. For c.835G>A (Asp279Asn), two primer pairs F and r-835 and R-BamHI and f-835, were used in the first PCR step. Primers F and R-BamHI were used in the second step. For c.2783G>A (Gly928Glu), primers F-BamHI and r-2783 and R and f-2783 were used in the first PCR step. Primers F-BamHI and R were used in the second step. For each mutation, amplification products in the first step were cleaned (Axygen, CA, USA), mixed and used as the template in the second PCR reaction. All final PCR amplifications were ligated into the digested pEGFP-N1 vector using ClonExpress.

Recombinant plasmids pEGFP-GC1, pEGFP-Asp279Asn and pEGFP-Gly928Glu were transformed into Escherichia coli DH5a cells. DNA was prepared by using a plasmid DNA purification kit from Macherey-Nagel following the manufacturer's instructions. Sanger sequencing was used to verify sequences.

\section{Cellular localization of wt and mutant ROS-GC1 recombinant plasmids}

pEGFP-N1, pEGFP-GC1, pEGFP-Asp279Asn and pEGFP-Gly928Glu were transfected into HeLa cells using the PolyJet Reagent (SigmaGen, MD, USA). After 36 h, cells were washed and fixed in 4\% paraformaldehyde. The antibody anti-Na ${ }^{+} / \mathrm{K}^{+}$-ATPase (1:100, HuaAn Biotechnology Co., Ltd., Hangzhou, China) was used to identify the plasma membrane of HeLa cells. Details about the process have been described previously [10]. In HeLa cells, wt and mutant ROS-GC1 were co-express with EGFP, and the localization of pEGFP-N1, pEGFP-GC1, pEGFPAsp279Asn and pEGFP-Gly928Glu was acquired by observing EGFP at 488nm excitation wavelength using a Nikon A1R.

\section{Western blotting}

Proteins from transfected HeLa cells were electrophoresed on a $12 \%$ sodium dodecyl sulfate-polyacrylamide gel (SDS-PAGE) and transferred to polyvinylidene fluoride (PVDF) membranes (Millipore, MA, USA), and then incubated with the primary antibodies, anti-GFP (1:1000, Proteintech, Wuhan, China) or anti-glyceraldehyde 3 phosphate dehydrogenase (GAPDH) (1:5000, Abcam, Cambridge, UK) overnight at $4{ }^{\circ} \mathrm{C}$. The PVDF membranes were washed with PBS and incubated with fluorescent secondary antibodies (1:1000, Abbkine, Wuhan, China) for $2 \mathrm{~h}$ at room temperature. The protein bands were visualized using an Odyssey Imager (Li-Cor Biosciences, NE, USA). GAPDH was used as the standard.

\section{Validation of cGMP quantitation by HPLC-MS/MS}


HeLa cells were transfected with pEGFP-N1, pEGFP-GC1, pEGFP-Asp279Asn and pEGFP-Gly928Glu. After 36 h, cells were collected from $100 \mathrm{~mm}$ plates and washed three times with PBS. The supernatant was removed carefully and $300 \mathrm{~mL}$ of ice-cold extraction medium (acetonitrile/methanol/water, 2/2/1 v/v/v) was added to each tube. Twentyfive $\mathrm{ng} / \mathrm{mL}$ Tenofovir (TNF) was added as the internal standard. After dissolving, the sample was frozen immediately in liquid nitrogen for $30 \mathrm{~s}$ to terminate cGMP metabolism, and this was followed by incubation in a 37 ${ }^{\circ} \mathrm{C}$ water bath for $60 \mathrm{~s}$. After repeating 6 times, samples were heated at $98^{\circ} \mathrm{C}$ for $20 \mathrm{~min}$. Samples were cooled on ice and centrifuged at $20,000 \times g$ at $4^{\circ} \mathrm{C}$ for $10 \mathrm{~min}$. The supernatant was transferred into a new tube and the nondissolved residue was extracted two more times with $400-\mu \mathrm{L}$ extraction medium. After evaporation, residues were collected and dissolved in water for further analysis.

The cGMP concentrations were analyzed via HPLC-MS/MS. The samples were applied to an HPLC utilizing an ACQUITY CSH-C18 column $(1.7 \mu \mathrm{m}, 2.1 \times 100 \mathrm{~mm}$ column, Waters, Ireland). The binary pump system supplied two eluents for chromatographic analysis, eluent A (10 mM formic acid) and eluent B (acetonitrile). The flow rate was $0.3 \mathrm{~mL} / \mathrm{min}$. Analyte detection was conducted on a sensitive triple quadrupole mass spectrometer (Waters TQ-XS, USA). Nitrogen was used as the collision gas. One-hundred ng/mL cGMP (G7504, Sigma, Germany) was used as the standard. All processes were referenced to the method described previously [11]. cGMP concentrations presented as means \pm SEM are based on three independent experiments. $P$-values were calculated by means of the independent sample T-test.

\section{Bioinformatics analysis}

All sequences were analyzed by Mutation Surveyor software and aligned to the NCBI nucleotide sequence of GUCY2D (NG_009092.1). The pathogenicity of mutations was evaluated using the in silico predictors SIFT (http://sift.jcvi.org/), PolyPhen-2 (http://genetics.bwh.harvard.edu/pph2/) and Mutation Taster (http://www.mutationtaster.org/). Computational modeling of the mutant ROS-GC1 by Chimera (PDB ID: 1AWL) was carried out to study the effect of the Gly928Glu mutation on the three-dimensional (3D) structure of ROS-GC1.

\section{Results}

\section{Clinical description of the proband}

The proband (III-2) was a five-year-old girl who showed the disorder visually but could perceive light in a dark room. The proband displayed the classic signs of oculo-digital symptoms. Ophthalmological examination revealed pendular nystagmus, roving eye movements, macular "colobomas" and optic disc abnormalities. Additionally, dim retina and salt-and-pepper pigmentation were found in the fundus. The results of flash visual evoked potential (VEP) showed that the latent period of binocular P-waves was severely prolonged and the amplitudes were severely reduced. The electroretinogram (ERG) results showed that the latent period and the amplitude of binocular scotopia rod response $b$-wave, mixed response a-wave and mixed response b-wave were flat. With an attenuated photopic $30-\mathrm{Hz}$ flicker, the full-field ERG (ffERG) showed a worsening delay of the cone-dependent response. The latent period and amplitude of the binocular photopic cone response a-wave and b-wave were flat. Clinical examination of the parents confirmed that neither was affected. 


\section{GUCY2D mutation analysis}

In order to discover the pathogenesis of the proband, targeted-NGS was performed using Agilent SureSelect All Exome Kit with Hiseq2000 sequencer. After analyzing the sequencing results of targeted-NGS, two novel mutations, c.139_139delC and c.2783G>A, in the GUCY2D gene were identified in the proband (III-2). Sanger sequencing confirmed the presence of c.139_139delC and c.2783G>A in other family members. The sequencing results showed that II-2 and II-3 are heterozygous mutation carriers that possess c.139_139delC and c.2783G>A, respectively (Fig. 1B1,2).

III-3 was described as an LCA1 patient (Fig. 1A). Sanger sequencing was used to determine the GUCY2D gene sequences of the suspected sibling of the patient (III-4) and parents (II-7 and II-8). The results showed that II-7 is a c. 2783G >A mutation carrier, and II-8 possesses another novel mutation c.835G>A (Fig. 1B3). The sibling (III-4) possessed no GUCY2D mutation. In addition, all heterozygous mutation carriers showed no clinical symptoms.

\section{Prediction of the pathogenic effect of ROS-GC1 mutations}

Ala49Profs*36, Asp279Asn and Gly928Glu are located in the LS, ECD and CCD of ROS-GC1, respectively (Fig. 2C). After analyzing the three mutations by SIFT, PolyPhen-2 and Mutation Taster, we propose that c.835G>A (SIFT: damaging, score: 0.01; PloyPhen-2: probably damaging, score: 1.00; Mutation Taster: disease-causing), c.2783G>A (SIFT: damaging, score: 0.00; PloyPhen-2: probably damaging, score: 1.00; Mutation Taster: disease causing) and c.139_139delC (Mutation Taster: disease-causing) are disease-causing mutations. Additionally, multiple sequence alignments showed that aspartic acid and glycine at positions 279 and 928 are highly conserved across species (Additional Fig. 1), indicating that Asp279 and Gly928 play important roles in ROS-GC1 and that mutations to these two residues are likely to cause ROS-GC1 dysfunction.

The high-resolution 3D structure of full-length ROS-GC1 remains unsolved. The theoretical model (PDB ID: 1AWL), which contains 158 amino acids from 871 to 1028 was used to study the impact of c.2783G>A (Gly928Glu) on the 3D structure of ROS-GC1. Although it is theoretical and not validated, 3D modeling analysis showed that Glu928 directly interacts with GTP (Fig. 2) and this change may reduce the size of the catalytic core, leading to a change in the relative position of GTP and the catalytic core. Furthermore, this mutation likely hampers ligand binding and reduces the catalytic activity of ROS-GC1.

\section{Localization of wt and mutant ROS-GC1}

ROS-GC1 is a member of the membrane guanylyl cyclase family, the correct localization of ROS-GC1 is critical for the synthesis of cGMP. In this study, immunofluorescence was used to confirm the localization of wt and mutant ROS-GC1. pEGFP-N1, pEGFP-GC1, pEGFP-Asp279Asn and pEGFP-Gly928Glu were transfected into HeLa cells after verification by Sanger sequencing (Additional Fig. 2). In Hela cells, ROS-GC1 was found to be present in cell membranes and cytoplasm (endoplasmic reticulum within cytoplasm [7]). The locations of Asp279Asn and Gly928Glu were the same as those found in the wt ROS-GC1. (Fig. 3, top panels). The anti-Na ${ }^{+} / \mathrm{K}^{+}$-ATPase antibody was used as a specific marker of the plasma membrane (Fig. 3, second panels). 


\section{Catalytic features of wt and mutant ROS-GC1}

HPLC-MS/MS was used to analyze cGMP concentrations in HeLa cells. The cGMP concentration in cells transfected with pEGFP-N1 was undetectable. In contrast, the cGMP concentration in cells transfected with pEGFPGC1 was about $3.631 \mathrm{pmol} / \mathrm{mL}$. Compared with wt, cells transfected with pEGFP-Asp279Asn or pEGFP-Gly928Glu showed significantly lower concentrations of cGMP (Fig. 4A), implying that Asp279Asn and Gly928Glu significantly reduced the catalytic activity of ROS-GC1. In addition, Gly928Glu disrupted the catalytic activity of ROS-GC1 more severely than the other mutants, which is consistent with the bioinformatics analysis. Moreover, all missense mutations did not affect the expression level of ROS-GC1 (Fig. 4B).

\section{Discussion}

Twenty known genes associated with LCA have been identified. The large number of genes and exons and the lack of mutational hot spots make individual screening for disease-causing mutations by Sanger sequencing difficult and expensive. However, NGS technologies provide more convenient and effective strategies to screen for pathogenic mutations in genes [12-14]. In this study, a family with one LCA proband was analyzed. Targeted-NGS and Sanger sequencing were combined to identify three mutations (c.139_139delC, c.2783G>A and c.835G>A) in the GUCY2D gene in this pedigree, and all mutations have yet to be documented by the 1000 Genomes Project. According to the description, there are two suspected patients in this pedigree, III-1 and III-3. However, both of them died at a very young age, thereby we only verified the GUCY2D gene mutation in II-7 and II-8. Additionally, in this pedigree all the heterozygous mutation carriers showed no clinical symptoms.

The novel disease-causing mutation c.139_139delC (Ala49Profs*36) carried by II-1 and putatively by II-2 and III-3 generates a truncated protein (83 amino acids), containing only a part of the LS (Fig. 2C). As all domains of ROSGC1 are presumably absent, there should be no enzymatic activity for this ROS-GC1 mutant and thus no cGMP production. Thus, the absence of sufficient levels of cGMP results in photoreceptor cell polarization, which finally leads to vision loss.

Multiple sequence alignments indicated that aspartic acid and glycine at positions 279 and 928 are highly conserved across species, indicating that these residues are important for protein function. This is consistent with previous observations that mutations located in the ECD domain decrease cGMP production and result in LCA1 [15]. The Asp279Asn mutation of this reported family decreased ROS-GC1 catalytic activity significantly; however, this mutation does not alter the cellular localization and the expression level of ROS-GC1. Mutant c.2783G $>A$ (Gly928Glu) is located in the pocket like catalytic domain of ROS-GC1 [16]. Bioinformatics analysis was used to estimate the effects of Gly928Glu on the catalytic activity of ROS-GC1. In contrast to Gly928, Glu928 directly interacts with the substrate GTP. Alteration of the catalytic core pocket by the Gly $928 \mathrm{Glu}$ mutation perturbs the catalytic activity of ROS-GC1. HPLC-MS/MS was used to further characterize the effect of the missense mutations on ROS-GC1 activity, and therefore provide additional experimental evidence of the effects of the mutations on ROS-GC1 activity. Results from HPLC-MS/MS showed that mutations Asp279Asn and Gly928Glu decreased ROSGC1 activity significantly, especially Gly928Glu, consistent with the 3D-modeling and bioinformatics analysis by SIFT, PolyPhen-2 and Mutation Taster. In contrast, Gly928Glu shares similar characteristics as wt ROS-GC1, including cellular localization and the level of protein expression.

In this study, the endogenous concentration of cGMP in HeLa cells, which were transfected with pEGFP-N1, is too low to be measured. This phenomenon can be observed in other studies of GUCY2D mutations, such as HEK293 
cells expressed mutant ROS-GC1 (A710V and P873R) showed undetectable cGMP concentrations, indicating that endogenous CGMP concentration exerted no contribution to CGMP levels. Although GUCY2D gene encodes a retinaspecific protein, use other cell lines to investigate the localization and enzymatic activity of ROS-GC1 is feasible, for example, HEK293 cells were widely used to study the cellular localization of ROS-GC1 [7, 10]. Additionally, HEK293, COS-7 and HeLa cells had been applied in the ROS-GC1 activity assays $[17,18]$.

\section{Conclusions}

In this study, we found three novel mutations (c.139_139delC, c.2783G>A and c.835G>A) in the GUCY2D gene. Mutation c.139_139delC results in a truncated protein that lacks all functional domains of ROS-GC1. Although mutations c. $835 \mathrm{G}>\mathrm{A}$ and $\mathrm{c} .2783 \mathrm{G}>\mathrm{A}$ showed normal protein expression levels and subcellular localization, both mutations significantly reduced the catalytic activity of ROS-GC1. As an alternative to radioisotope labeling assays, HPLC-MS/MS was used herein to analyze cGMP concentrations. Compared with traditional methods, HPLC$\mathrm{MS} / \mathrm{MS}$ is more convenient and is an effective alternative to evaluate the cGMP concentration in cells.

\section{Abbreviations}

LCA: Leber congenital amaurosis; GUCY2D: guanylate cyclase 2D; ROS-GC1: guanylate cyclase protein 1; GCAPs: guanylate cyclase-activating proteins; CCD: cyclase catalytic domain; DD: dimerization domain; ECD: extracellular domain; JMD: juxtamembrane domain; KHD: kinase homology domain; LS: leader sequence; TM: transmembrane domain; HPLC-MS/MS: HPLC-coupled tandem mass-spectrometry; wt: wild type; NGS: next-generation sequencing.

\section{Declarations}

\section{Ethics approval and consent to participate}

All procedures performed were in accordance with the Declaration of Helsinki and approved by the ethical standards of the Institutional Review Board, Zhejiang University. Written informed consent was obtained from all individuals who participated in this study and the parent of children under 16.

\section{Consent to publish}

not applicable.

\section{Availability of data and materials}

The data used and analyzed in this study are available from the corresponding author.

\section{Competing of interests}

The authors report no conflict of interests.

\section{Funding}

The study was supported by grants from the National Natural Science Foundation of China $(31371271,81200662)$ and the Natural Science Foundation of Zhejiang Province (LZ14C060001, LY12H12010). In this research, the funding organization exerted no role in the design or conduct. 


\section{Authors' contributions}

P.Y. and Y.H.Y. designed the experiment, X.F., T.Y.W and J.H.S. performed the experiment and analyzed the data, Y.N.H., J.C. and X.M.W. recruited the proband and the family members, X.F. and T.Y.W. write the paper, Y.H.Y and M.Q. modified the paper. All the authors approved the final manuscript.

\section{Acknowledgments}

We thank the patient and his family for their cooperation and participation in the study. Additionally, we thank Liwen Bianji, Edanz Editing China (www.liwenbianji.cn/ac), for editing the English text of a draft of this manuscript.

\section{References}

1. Perrault I, Rozet JM, Gerber S, Ghazi I, Leowski C, Ducroq D, Souied E, Dufier JL, Munnich A, Kaplan J. Leber congenital amaurosis. Molecular genetics and metabolism 1999; 68:200-8.

2. Cremers FP, van den Hurk JA, den Hollander Al: Molecular genetics of Leber congenital amaurosis. Human molecular genetics 2002; 11:1169-76.

3. Kaplan J, Bonneau D, Frezal J, Munnich A, Dufier JL. Clinical and genetic heterogeneity in retinitis pigmentosa. Human genetics 1990; 85:635-42.

4. den Hollander Al, Roepman R, Koenekoop RK, Cremers FP. Leber congenital amaurosis: genes, proteins and disease mechanisms. Progress in retinal and eye research 2008; 27:391-419.

5. Chacon-Camacho OF, Zenteno JC. Review and update on the molecular basis of Leber congenital amaurosis. World journal of clinical cases 2015; 3:112-24.

6. Sharon D, Wimberg H, Kinarty Y, Koch KW. Genotype-functional-phenotype correlations in photoreceptor guanylate cyclase (GC-E) encoded by GUCY2D. Progress in retinal and eye research 2018; 63:69-91.

7. Zagel P, Koch KW. Dysfunction of outer segment guanylate cyclase caused by retinal disease related mutations. Frontiers in molecular neuroscience 2014; 7:4.

8. Gnirke A, Melnikov A, Maguire J, Rogov P, LeProust EM, Brockman W, Fennell T, Giannoukos G, Fisher S, Russ C, Gabriel S, Jaffe DB, Lander ES, Nusbaum C. Solution hybrid selection with ultra-longoligonucleotides for massively parallel targeted sequencing. Nature Biotechnology 2009;27: 182-189.

9. Ho Duy B, Zhytnik L, Maasalu K, Kandla I, Prans E, Reimann E, Martson A, Koks S. Mutation analysis of the COL1A1 and COL1A2 genes in Vietnamese patients with osteogenesis imperfecta. Human genomics 2016; $10: 27$.

10. Zagel P, Dell'Orco D, Koch KW. The dimerization domain in outer segment guanylate cyclase is a $\mathrm{Ca}(2)(+)$ sensitive control switch module. Biochemistry 2013; 52:5065-74.

11. Beckert U, Aw WY, Burhenne H, Forsterling L, Kaever V, Timmons L, Seifert R. The Receptor-Bound Guanylyl Cyclase DAF-11 Is the Mediator of Hydrogen Peroxide-Induced cGMP Increase in Caenorhabditis elegans [corrected]. PloS one 2013; 8:e72569.

12. Siu LL, Conley BA, Boerner S, LoRusso PM. Next-Generation Sequencing to Guide Clinical Trials. Clinical cancer research : an official journal of the American Association for Cancer Research 2015; 21:4536-44.

13. Nuytemans K, Vance JM. Whole exome sequencing. Rinsho shinkeigaku = Clinical neurology 2010; 50:952-55.

14. Ng SB, Buckingham KJ, Lee C, Bigham AW, Tabor HK, Dent KM, Huff CD, Shannon PT, Jabs EW, Nickerson DA et al. Exome sequencing identifies the cause of a mendelian disorder. Nature genetics 2010; 42:30-35. 
15. Tucker CL, Ramamurthy V, Pina AL, Loyer M, Dharmaraj S, Li Y, Maumenee IH, Hurley JB, Koenekoop RK. Functional analyses of mutant recessive GUCY2D alleles identified in Leber congenital amaurosis patients: protein domain comparisons and dominant negative effects. Molecular vision 2004; 10:297-303.

16. Liu Y, Ruoho AE, Rao VD, Hurley JH. Catalytic mechanism of the adenylyl and guanylyl cyclases: modeling and mutational analysis. Proceedings of the National Academy of Sciences of the United States of America 1997; 94:13414-19.

17. Gibb BJ, Wykes V, Garthwaite J. Properties of NO-activated guanylyl cyclases expressed in cells. British Journal of Pharmacology 2003 Jul;139(5):1032-40.

18. Wimberg H, Lev D, Yosovich K, Namburi P, Banin E, Sharon D, Koch KW. Photoreceptor Guanylate Cyclase (GUCY2D) Mutations Cause Retinal Dystrophies by Severe Malfunction of $\mathrm{Ca}^{2+}$-Dependent Cyclic GMP Synthesis. Frontiers in Molecular Neuroscience 2018 Sep 25;11:348.

\section{Tables}

Table 1 Primers for mutation screening. 


\begin{tabular}{|c|c|c|}
\hline Name & Sequence & Length $(\mathrm{bp})$ \\
\hline GUCY2D-exon1-F & GAAGCCAGGACAGATCCCAC & 322 \\
\hline GUCY2D-exon1-R & GGAGGCGTCAGGGGTCA & \\
\hline GUCY2D-exon2-1-F & GCCCCAGTTAGTCTTCCCAG & 550 \\
\hline GUCY2D-exon2-1-R & AGGGTTCACCGGACCCAC & \\
\hline GUCY2D-exon2-2-F & TGCTGCCCGAGCCTTG & 549 \\
\hline GUCY2D-exon2-2-R & TCCTCGCCACCCAGCA & \\
\hline GUCY2D-exon3-F & CAGGTAGGCTCCCTTGCAG & 496 \\
\hline GUCY2D-exon3-R & AGAGCTGCCAGTGGTTCTTT & \\
\hline GUCY2D-exon4-F & AACAGTGGATACCCTGGGC & 550 \\
\hline GUCY2D-exon4-R & GGGCATCGAAGACGGATTAC & \\
\hline GUCY2D-exon5-F & АTTCCCAGCCTCTCCCCTTT & 387 \\
\hline GUCY2D-exon5-R & ACTCTACCAGCCCACCAGAA & \\
\hline GUCY2D-exon6-F & TTCTGGTGGGCTGGTAGAGT & 464 \\
\hline GUCY2D-exon6-R & CCCGCCAGGAAAGTAGTCAG & \\
\hline GUCY2D-exon7-F & CCTGACTACTTTCCTGGCGG & 477 \\
\hline GUCY2D-exon7-R & TCCCTAGATCCTGTCTGGGC & \\
\hline GUCY2D-exon8-F & ATGGCTGTGAAGTGGATGGG & 423 \\
\hline GUCY2D-exon8-R & АТССТСССТСАСАТСТGССТ & \\
\hline GUCY2D-exon9-F & GAGAGCCCCCGTACATACCT & 487 \\
\hline GUCY2D-exon9-R & CGCCTCGATGGTGCAGATAC & \\
\hline GUCY2D-exon10-F & ATCAAGGTGTGTGTCTGGGG & 476 \\
\hline GUCY2D-exon10-R & CAGCTCAGGTACAAGTCCCG & \\
\hline GUCY2D-exon11-F & GGCCTATTTGCCAGGCTTTC & 476 \\
\hline GUCY2D-exon11-R & ACCTGCAGATGCCAGCTTT & \\
\hline GUCY2D-exon12-F & CTAGCAACCCCCTTCCACAC & 402 \\
\hline GUCY2D-exon12-R & AGCTGTCTCAGGTTGCTGAC & \\
\hline GUCY2D-exon13-F & ACGTGCCTCCTAATCGTGTC & 505 \\
\hline GUCY2D-exon13-R & GCTCAAAGTACTCGGGCTCC & \\
\hline GUCY2D-exon14-F & GCTGCTTACACAGATGCTGC & 440 \\
\hline GUCY2D-exon14-R & TAAGGGACAGGAGGTCTGGG & \\
\hline GUCY2D-exon15-F & CAGCTCAGTCCTTCCACTAGC & 468 \\
\hline GUCY2D-exon15-R & CGCACCCATTATCTCCACCA & \\
\hline
\end{tabular}




\begin{tabular}{lll} 
GUCY2D-exon16-17-F & AGAGGATGCACTTAACAAGGCT & 538 \\
GUCY2D-exon16-17-R & ATCTCGAGTCTGCGTGGAAC & \\
GUCY2D-exon18-F & CCCTGTCCTGAGGCACC & 365 \\
GUCY2D-exon18-R & CTCAGGGAAGGGGAATGGG & \\
GUCY2D-exon19-F & CGAGGGACCCCTGCCT & 393 \\
GUCY2D-exon19-R & ATTCCTGCAATGGCTGCTTC & \\
GUCY2D-exon20-F & TAGCTGGCAGAGCAGTGATG & 487 \\
GUCY2D-exon20-R & ACTTCCCCTCTTCAGGCCAT & \\
\hline
\end{tabular}

Table 2 Primers for construction of recombination plasmids.

\begin{tabular}{|c|c|c|}
\hline Primer & Sequence & $\begin{array}{l}\text { Restriction } \\
\text { Enzyme }\end{array}$ \\
\hline $\mathrm{F}$ & TCCGCTAGCGCTACCGGACTCAGATctcgagATGACCGCCTGCGCCCGCCGAGCGGGTGGGCTT & Xhol \\
\hline $\mathrm{R}$ & CTCGCCCTTGCTCACCATGGTGGCGaccggtGGTGAAGAGAACTGGCCCGGCCGCGCC & Agel \\
\hline$f-835$ & GGCTCCCTGGTCTTCCTGCCCTTCAACACGATCCACTACGCCTTGTCCCCA & \\
\hline r-835 & TGGGGACAAGGCGTAGTGGATCGTGTtGAAGGGCAGGAAGACCAGGGAGCC & \\
\hline R- & GGCGGAGAGGAAGGAGCCCCGGGCAggatccAGCATGTATGTGGCAAAAAGCCGGT & BamHI \\
\hline \multicolumn{3}{|l|}{ BamHI } \\
\hline F- & ACCGGCTTTTTGCCACATACATGCTggatccTGCCCGGGGCTCCTTCCTCTCCGCC & BamHI \\
\hline \multicolumn{3}{|l|}{ BamHI } \\
\hline$f-2783$ & CTACAAGGTGGAGACAATAGaGGACGCCTATATGGTGGCC & \\
\hline $\mathrm{r}-2783$ & GGCCACCATATAGGCGTCCtCTATTGTCTCCACCTTGTAG & \\
\hline
\end{tabular}

\section{Additional Table 1}

Additional Table 1 Screening scope of the targeted-NGS in eye diseases. 


\begin{tabular}{|c|c|}
\hline Disease name & Pathogenic \\
\hline Retinitis & ABCA4, AIPL 1, ARL6, BEST1, CA4, CERKL, C2orf71, CLRN1, DHDDS, NGA1, CNGB1, CRB1, \\
\hline pigmentosa & CRX, EYS, FAM161A, FSCN2, GUCA1B, IDH3B, IMPDH1, IMPG2, KLHL7, LRAT, MAK, MERTK, \\
\hline (including & NR2E3, NRL, PDE6A, PDE6B, PRCD, PDE6G, PROM1, PRPF3, PRPF31, PRPF6, PRPF8, \\
\hline autosomal & $P R P H 2, R B P 3, R D H 12, R G R, R H O, R L B P 1, R O M 1, R P 1, R P 2, R P 9, R P E 65, R P G R, S A G$, \\
\hline $\begin{array}{l}\text { dominant, } \\
\text { autosomal }\end{array}$ & SEMA4A, SNRNP200, TOPORS, TULP1, USH2A, ZNF513 \\
\hline recessive chain/X) & \\
\hline $\begin{array}{l}\text { Waardenburg } \\
\text { syndrome }\end{array}$ & EDNRB, MITF, PAX3, SNAI2, SOX10, EDN3 \\
\hline $\begin{array}{l}\text { x-linked juvenile } \\
\text { retinoschisis }\end{array}$ & RS1 \\
\hline $\begin{array}{l}\text { Crystalline retinitis } \\
\text { pigmentosa }\end{array}$ & CYP4V2 \\
\hline $\begin{array}{l}\text { Bothnia retinal } \\
\text { dystrophy }\end{array}$ & RLBP1 \\
\hline $\begin{array}{l}\text { Best macular } \\
\text { dystrophy }\end{array}$ & BEST1 \\
\hline Congenital aniridia & PAX6 \\
\hline $\begin{array}{l}\text { Albinism (non } \\
\text { syndromic) }\end{array}$ & GPR143, MITF, OCA2, TYR, OCA2, TYRP1, SLC45A2 \\
\hline $\begin{array}{l}\text { Leber congenital } \\
\text { amaurosis }\end{array}$ & $\begin{array}{l}\text { GUCY2D, RPE65, SPATA7, AIPL1, LCA5, RPGRIP1, CRX, CRB1, NMNAT1, CEP290, IMPDH1, } \\
\text { RD3, RDH12, LRAT, TULP1, KCNJ13 }\end{array}$ \\
\hline $\begin{array}{l}\text { Bardet Biedl } \\
\text { syndrome }\end{array}$ & $\begin{array}{l}\text { BBS1, BBS2, ARL6, BBS4, BBS5, MKKS, BBS7, TTC8, BBS9, BBS10, TRIM32, BBS12, MKS1, } \\
\text { INPP5E }\end{array}$ \\
\hline Monochromasia & CNGA3, CNGB3, GNAT2, PDE6C , PDE6H \\
\hline $\begin{array}{l}\text { Protanopia } \\
\text { anerythrochloropsia }\end{array}$ & OPN1LW, OPN1MW \\
\hline Joubert syndrome & $\begin{array}{l}\text { AHI1, ARL13B, CC2D2A, CEP290, KIF7, INPP5E, NPHP1, OFD1, RPGRIP1L, TMEM216, } \\
\text { TMEM67 }\end{array}$ \\
\hline $\begin{array}{l}\text { Senior-Loken } \\
\text { syndrome }\end{array}$ & NPHP1, NPHP4, IQCB1, CEP290, SDCCAG8 \\
\hline Usher syndrome & $\begin{array}{c}\text { CDH23, CLRN1, DFNB31, GPR98, MYO7A, PDZD7, PCDH15, USH2A, USH1C, USH1G } \\
\text { Page } 13 / 17\end{array}$ \\
\hline
\end{tabular}


Age-related macular $\quad A B C A 4, T L R 4, C S T 3, C F H, H T R A 1, C 3$

degeneration

Cone dystrophy

CACNA2D4, GUCA1A, KCNV2, PDE6H, PDE6C

Cone-rod dystrophy

ABCA4, ADAM9, AIPL1, BEST1, CACNA1F, CDHR1, CERKL, CRX, GUCA2A, GUCY2D,

PITPNM3, PROM1, RAX2, RIMS1, RLBP1, RPGR, RPGRIP1, SEMA4A, UNC119

Nyctalopia

CACNA1F, CABP4, GNAT1, GPR179, GRK1, GRM6, NYX, PDE6B, RHO, SAG, SLC24A1,

TRPM1

\section{Additional Figure Legends}

Additional Fig. 1 Multiple sequence alignment of ROS-GC1 from different species reveals that codon 279 and 928 where the mutations Asp279Asn and Gly928Glu occurred, are located within highly conserved regions.

Additional Fig. 2 Sequencing results of wt and mutant GUCY2D. Sanger sequencing was used to verify the sequences of the recombinant plasmids pEGFP-GC1, pEGFP-Ala49Profs*36, pEGFP-Asp279Asn and pEGFPGly928Glu.

\section{Figures}

A

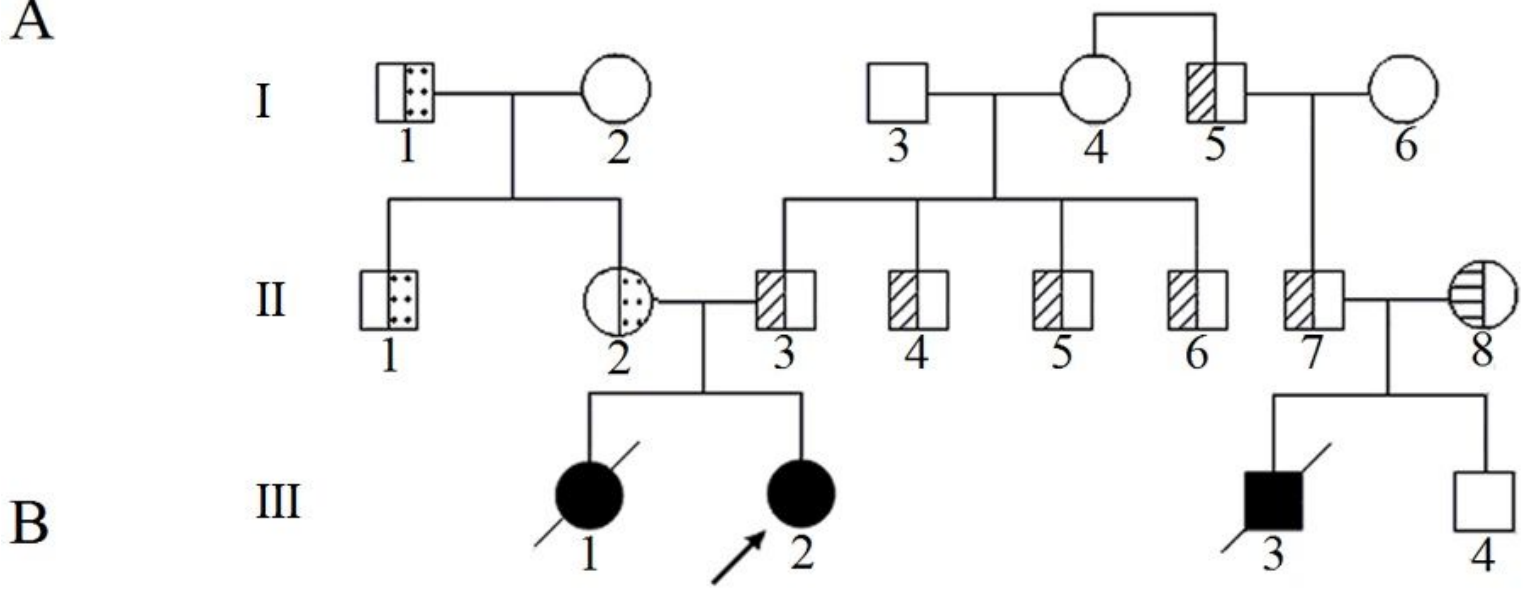

1 A G C C C C C C C C T C T 2 ACAATAGG GA C C

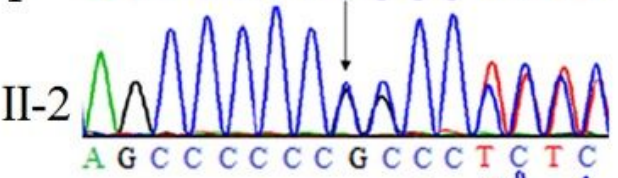

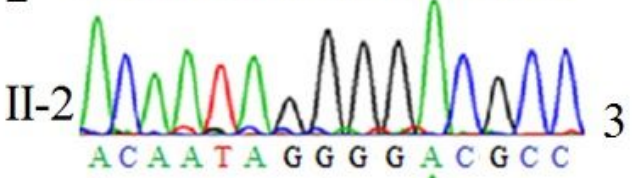

II-3 WMDMNANA A G C C C C C C C C C T C T C III-2LWOMánlows

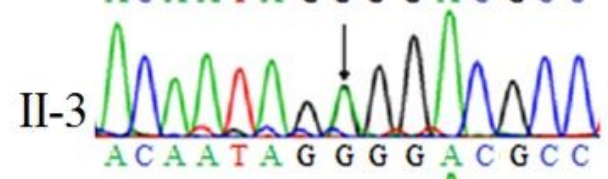
3 G C C C T T C A ACACGA T II-8) NMAn

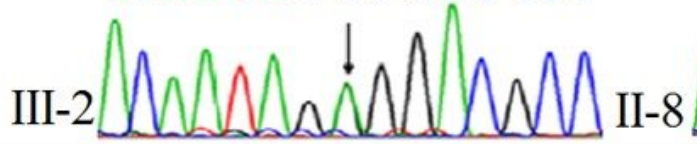

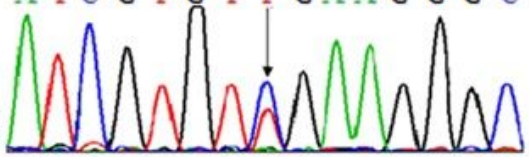

Figure 1 
Brief information of GUCY2D mutations in the LCA1 family. A: Pedigree of the LCA1 family. $\nearrow$ represents the proband, $\square$ represents the normal male, represents the normal female, crossed symbols represent the deceased subject. and denotes patients, the half-shaded icons denote the mutation carriers as =c.139_139delC, $=c .2783 \mathrm{G}>\mathrm{A}$, and $=\mathrm{c} .835 \mathrm{G}>\mathrm{A}$. B: 1 : The sequencing results of the family show the mutation c.139_139delC, indicated by the arrows. 2: The sequencing results of the family show the mutation c. $2783 \mathrm{G}>\mathrm{A}$, indicated by the arrows. 3: The sequencing result of the family member II-8 with mutation $\mathrm{c} .835 \mathrm{G}>\mathrm{A}$; forward sequencing result (top), reverse sequencing result (bottom).
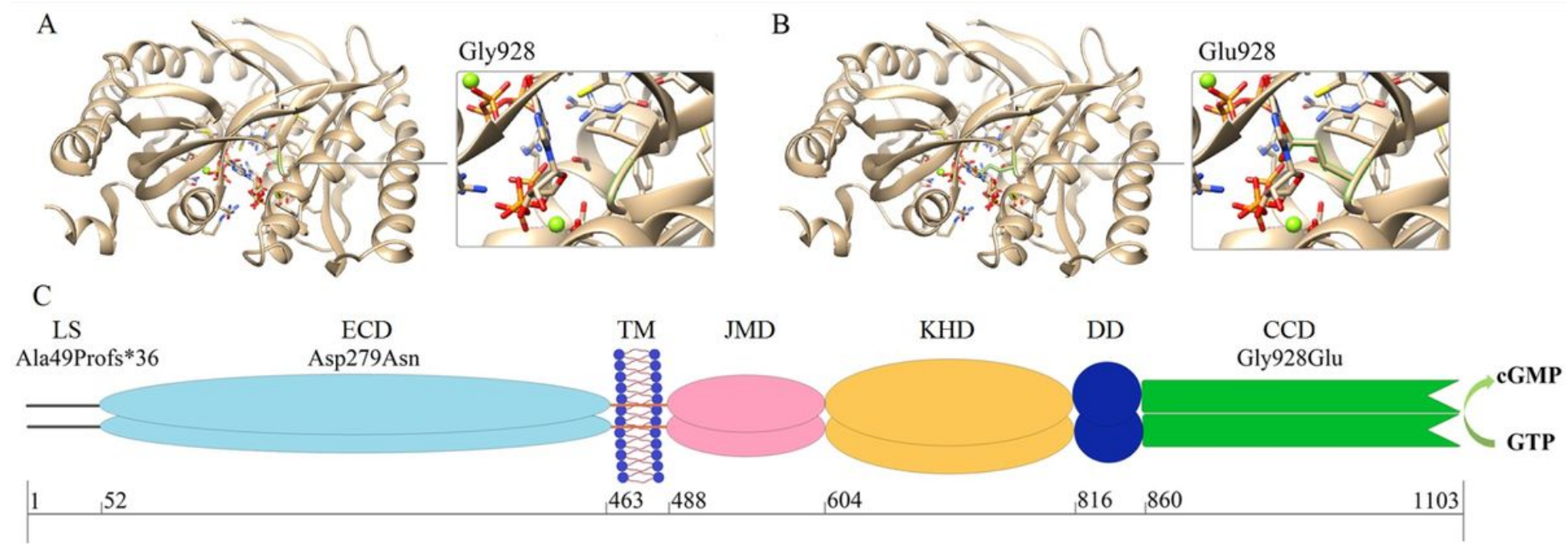

\section{Figure 2}

The protein domains and Modeling analysis of mutation Gly928Glu. A: 3D-modeling analysis of the Gly928. B: 3Dmodeling analysis of the Glu928. Green line indicates ROS-GC1 residue. C: ROS-GC1 domains and location of Ala49Profs*36, Asp279Asn and Gly928Glu. 


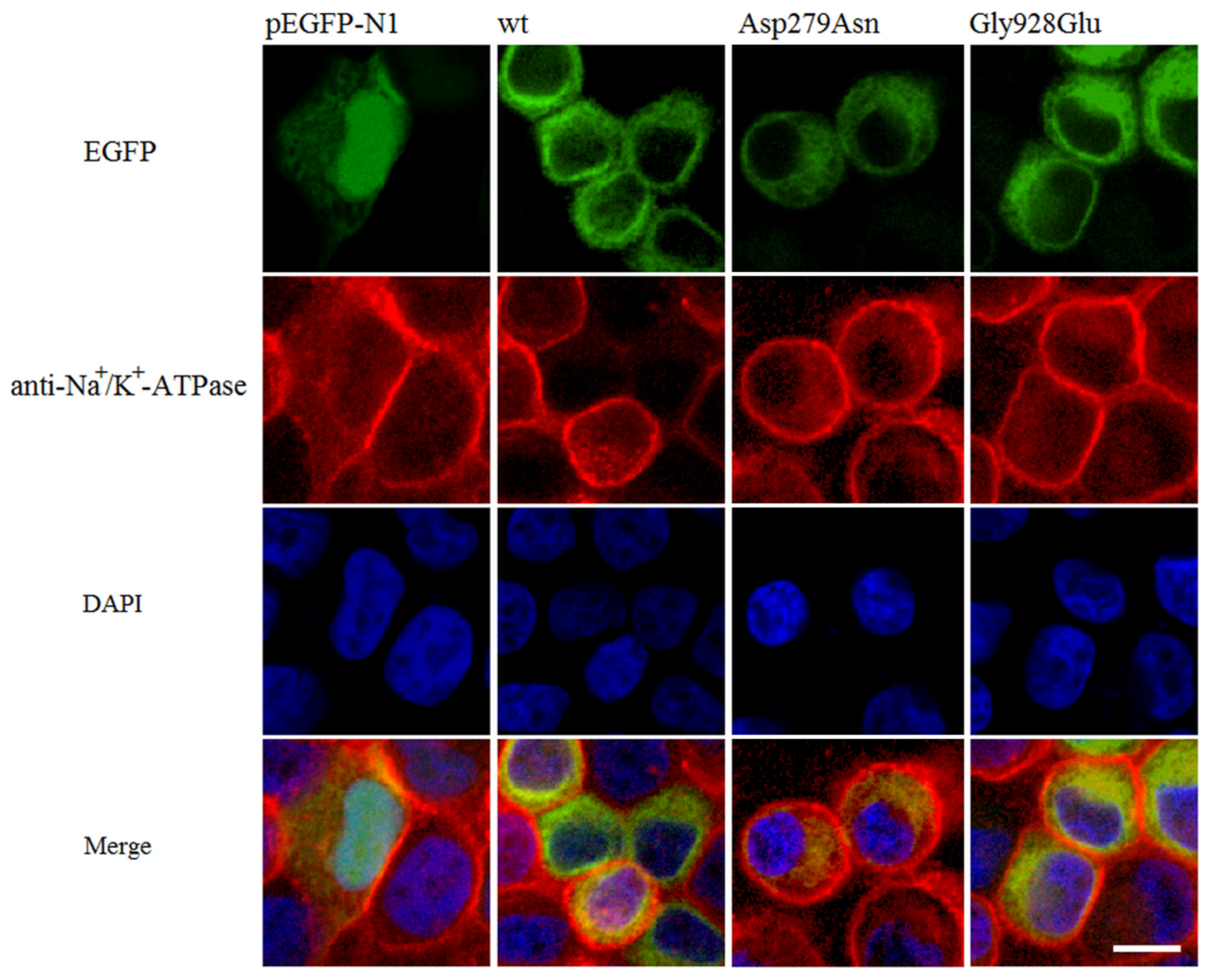

Figure 3

Cellular localization of wt and mutant ROS-GC1 in HeLa cells. ROS-GC1 wt and mutants were co-expressed with EGFP. Immunostaining from top to bottom: EGFP excited at 488nm (top panels), anti-Na+/K+-ATPase (second panels) as the cell membrane marker, DAPI (third panels) as the nucleus marker, and overlay images (bottom panels). The scale bar is $10 \mu \mathrm{m}$. 


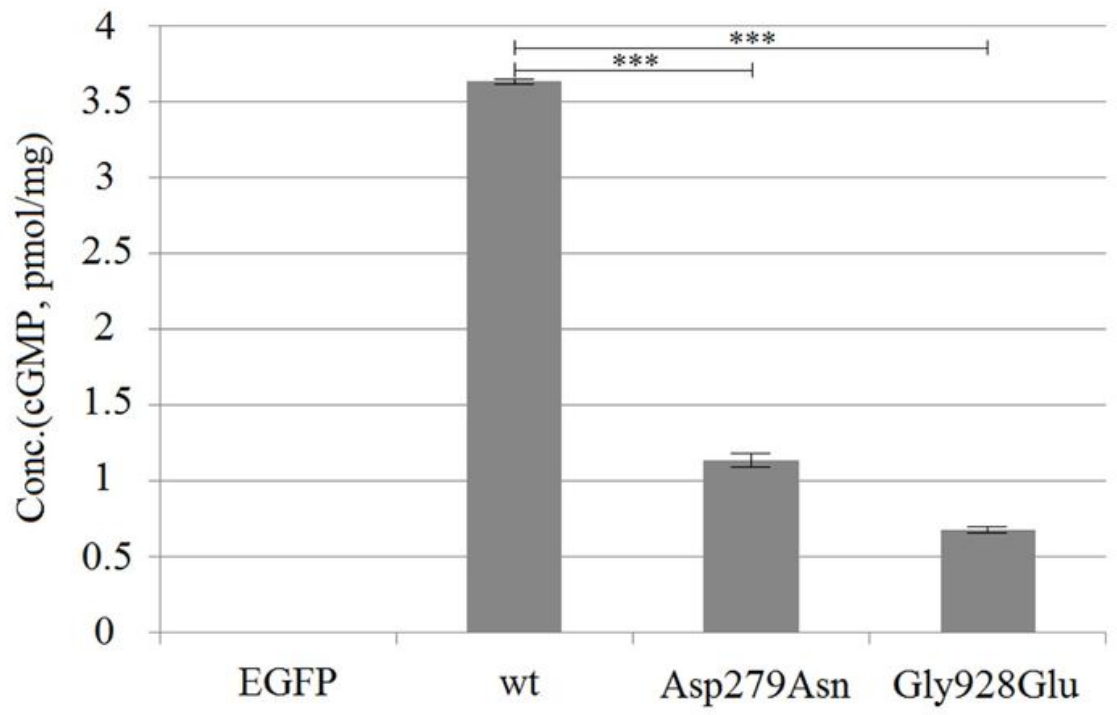

B anti-EGFP anti-GAPDH

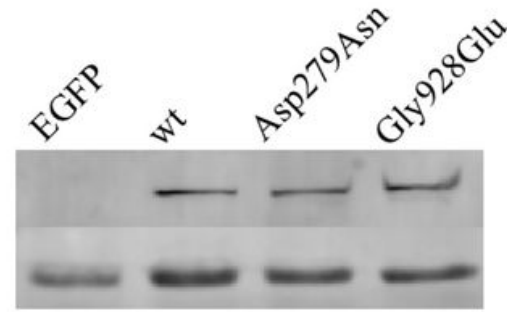

\section{Figure 4}

cGMP concentrations and ROS-GC1 expression levels in HeLa cells. A: Transfected HeLa cells with pEGFP-N1, pEGFP-GC1, pEGFP-Asp279Asn and pEGFP-Gly928Glu. Cells solution was collected and used to estimate cGMP concentrations. P-values were calculated by means of independent sample T-test. $* \star *$ : $p$ value $\leq 0.001$. B: Western blot of HeLa cells expressing human EGFP, wt and mutant ROS-GC1.

\section{Supplementary Files}

This is a list of supplementary files associated with this preprint. Click to download.

- AdditionalFigure1.jpg

- AdditionalFigure2.jpg 